\title{
Efficient Hybrid Block Method For The Numerical Solution Of Second-order Partial Differential Problems via the Method of Lines
}

\author{
Olumide O. Olaiya ${ }^{\mathrm{a}}$, Razaq A. Azeez ${ }^{\mathrm{b}}$, Mark I. Modebei ${ }^{\mathrm{a}}$ \\ ${ }^{a}$ Department of Mathematics Programme, National Mathematical Centre, Abuja, Nigeria \\ ${ }^{b}$ Department of Mathematics, University of Abuja, Abuja, Nigeria
}

\begin{abstract}
This study is therefore aimed at developing classes of efficient numerical integration schemes, for direct solution of second-order Partial Differential Equations (PDEs) with the aid of the method of lines. The power series polynomials were used as basis functions for trial solutions in the derivation of the proposed schemes via collocation and interpolation techniques at some appropriately chosen grid and off-grid points the derived schemes are consistent, zero-stable and convergent. the proposed methods perform better in terms of accuracy than some existing methods in the literature.
\end{abstract}

DOI:10.46481/jnsps.2021.141

Keywords: Initial Value Problem, Boundary Value Problem; Block method, Linear Multistep Method, Hybrid method, method od lines

Article History :

Received: 02 October 2020

Received in revised form: 10 December 2020

Accepted for publication: 23 January 2021

Published: 27 February 2021

(C)2021 Journal of the Nigerian Society of Physical Sciences. All rights reserved. Communicated by: T. Latunde

\section{Introduction}

In this work, we consider the second-order PDE of the form

$$
A \frac{\partial y^{2}}{\partial x^{2}}+B \frac{\partial y^{2}}{\partial t^{2}}+\mathrm{C} \frac{\partial y}{\partial t}=0, x \in[\mathrm{a}, \mathrm{b}], \mathrm{t}>0
$$

With any of the following initial-boundary conditions

$$
\begin{aligned}
& y\left(x, \alpha_{1}\right)=\xi_{1}, \quad y\left(x, \alpha_{2}\right)=\xi_{2}, \\
& y\left(\beta_{1} t\right)=\zeta_{1}, \quad y\left(\beta_{2}, t\right)=\zeta_{2}, \\
& y\left(x, \alpha_{1}\right)=\xi_{1}, \quad y\left(\beta_{2}, t\right)=\zeta_{2}
\end{aligned}
$$

Email address: olaiyaolumide. o@gmail.com (Olumide O. Olaiya )
, where $\alpha_{i}, \beta_{i}, \zeta_{i}, \xi_{i}, i=1,2, a, b, A, B, C$ are all real constants and $A \neq 0 . y \in C^{2}[a, b] \times[c, d], \quad x \in(a, b), \quad t \in(c, d)$. Second-order PDEs can either be of Laplacian, Poisson forms which could either be heat or waveform of equations. They find their applications in numerous areas of human endeavours, especially in mathematical sciences and engineering.

The developed differential equations require solutions, either in closed form (analytic form) or in numerical forms. In most cases, closed-form of solutions is rare to come by as there exist limited methods for solving such models in the form of differential equations. This brings to light the use of numerical methods/techniques to solve the modelled problem.

Numerical techniques are numerous and the types know no bounds. They include; The Euler method, Runge-Kutta methods, linear Multistep method, shooting method, Finite difference method, finite element methods, e.g Galerkin method, 
Spectral element method. Other methods are; Spectral method base on Fourier transformation; Method of lines reduces the PDE to a large system of the ordinary differential equation (ODE) Boundary Element Method (BEM) based on transforming a PDE to an integral equation on the boundary of the domains and it is popular in computational fluid dynamics, the list is endless. Authors who have worked extensively on numerical methods for approximation of solution of a differential equation include but no limited to Brugnano and Trigiante [1], Onumanyi et al. [2, 3], Jator [4, 5], Fatunla [6], Yusuph and Onumanyi [7], Siraj-ul-Islam, et al.[8], Adewale et al. [9].

We adopt the method of lines approach which is commonly used for solving time-dependent partial differential equations (PDEs), whereby the spatial derivatives are replaced by finite difference approximations see Ngwane and Jator [10].

The Method of Lines (MOLs) allows the conversion of PDEs into ODEs by complete or partial discretisation of the independent variables resulting in algebraic equations. If partial discretization is carried out and with only one remaining independent variable, then this results in the system of ODEs which is an approximation of the original PDE. Thus, one of the underscored features of the MOL is the use of existing, and generally well established, numerical methods for ODEs, for more literature on this approach see Brugnano and Trigiante [1], Ramos and Vigo-Aguiar [11], Cash [12] and Jator and Li [13].

\section{Derivation of the Method}

Second order ordinary differential equation of the form is considered

$$
y^{\prime \prime}=f(x, y)
$$

subject certain conditions, where $a, b$ are real numbers, $f$ is a continuous function on $(a, b)$ and $y \in C^{2}[a, b]$. A 2-step block methods for the problem of the form (1) is considered.

The grid points given by $x_{n}, x_{n+1}=x_{n}+h, x_{n+2}=x_{n}+2 h$, are considered for solving the problem in (1) on the interval $\left[x_{n}, x_{n+2}\right]$. We assume a trial solution $y(x)$ of (1) by a polynomial $p(x)$ given by

$$
y(x) \simeq p(x)=\sum_{i=0}^{m-1} a_{i} x^{i}
$$

which on differentiating yields

$$
y^{\prime \prime}(x) \simeq p^{\prime \prime}(x)=\sum_{i=2}^{m-1} i(i-1) a_{i} x^{i-2}
$$

with the $a_{i} \in \mathbb{R}$ real unknown parameters to be determined. and $m=r+s ; r$ is the number of interpolation points and $s$ is the number of collocation points.

\subsection{Specification of the method}

In this work the interval of integration considered is $\left[x_{n}, x_{n+2}\right]$, we thus consider two different categories of off-set points viz-aviz the points $x_{\frac{i}{3}}$, for $i=1,2,4,5$ and $x_{\frac{i}{4}}$, for $i=1,2,3,5,6,7$.

\subsubsection{Case 1}

Here, we consider the specification where the off-set points are $x_{\frac{i}{3}}$, for $i=1,2,4,5$. interpolating (2) at the points $x_{\frac{i}{3}}$, for $i=1,2$ implies $r=2$ and collocating (3) at points $x_{\frac{i}{3}}$, for $i=0(1) 6$ implies $s=7$ so that (2) and (3) becomes

$$
y(x) \simeq p(x)=\sum_{i=0}^{8} a_{i} x^{i}=a_{0}+a_{1} x+a_{2} x^{2}+\cdots+a_{8} x^{8}
$$

which on differentiating twice yields

$$
y^{\prime \prime}(x) \simeq p^{\prime \prime}(x)=\sum_{i=2}^{8} i(i-1) a_{i} x^{i-2}=2 a_{2}+6 a_{3} x+12 a_{4} x^{2}+\cdots+56 a_{8} x^{6}
$$


From the imposed collocation condition, the following system of algebraic equation is obtained

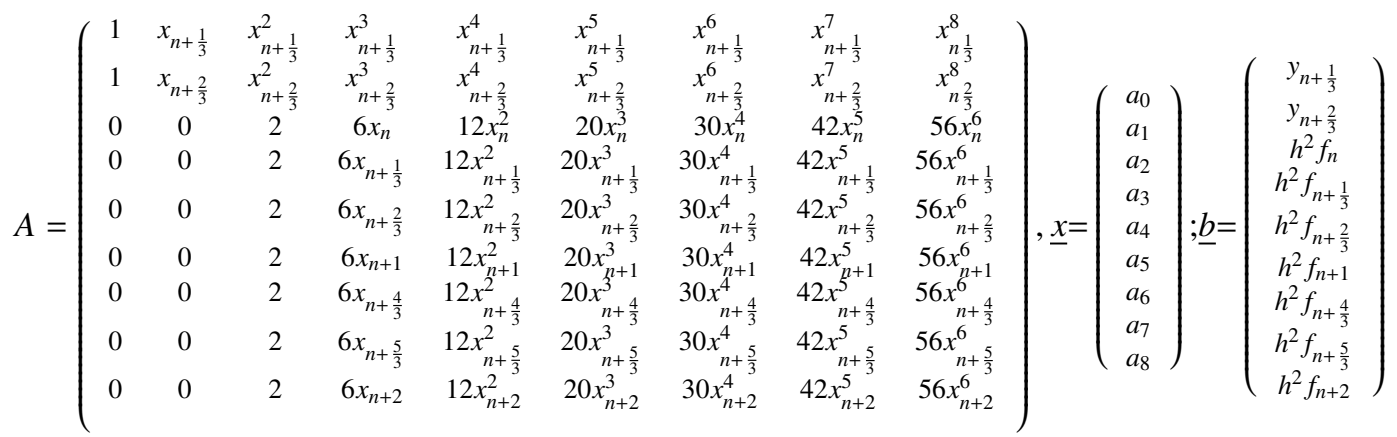

$$
\begin{aligned}
& \underline{e}=\left(1, x, x^{2}, x^{3}, x^{4}, x^{5}, x^{6}, x^{7}, x^{8}\right)^{T}
\end{aligned}
$$

Where $y_{n} \approx y\left(x_{n}\right), f_{n} \approx f\left(x_{n}, y_{n}, y_{n}^{\prime}\right)$.

Hence, we state the following theorem without proof.

Theorem 2.1. [14] Let (4) and (5) be satisfied, then the 2-step continuous linear hybrid multistep method is equivalent to the equation

$$
y(x)=\underline{b}^{T}\left(\mathbf{A}_{k}^{-1}\right)^{T} \underline{e}
$$

where $\underline{b}, \mathbf{A}$ and $\underline{e}$ are as defined above.

Applying the above theorem, the following continuous hybrid method is derived

$$
y(x)=\sum_{i=1}^{2} \alpha_{\frac{i}{3}} y_{n+\frac{i}{3}}+h^{2} \sum_{j=0}^{2} \beta_{\frac{i}{3}} f_{n+\frac{i}{3}}
$$

where $\alpha$ and $\beta$ are function of $t$ given as

$$
\begin{aligned}
& \alpha_{\frac{1}{3}}=-3 t+2, \quad \alpha_{\frac{2}{3}}=3 t-1 \\
& \beta_{0}=h^{2}\left[\frac{21}{32} t^{6}-\frac{459}{4480} t-\frac{27}{160} t^{7}+\frac{1}{2} t^{2}+\frac{81}{4480} t^{8}+\frac{863}{108894}-\frac{49}{40} t^{3}-\frac{441}{320} t^{5}+\frac{203}{120} t^{4}\right] \\
& \beta_{\frac{1}{3}}=h^{2}\left[\frac{-243}{2240} t^{8}+\frac{27}{28} t^{7}-\frac{279}{80} t^{6}+\frac{261}{40} t^{5}-\frac{261}{40} t^{4}+3 t^{3}-\frac{27623}{60480} t+\frac{8999}{90720}\right] \\
& \beta_{\frac{2}{3}}=h^{2}\left[\frac{243}{896} t^{8}-\frac{513}{224} t^{7}+\frac{1233}{160} t^{6}-\frac{4149}{320} t^{5}+\frac{351}{32} t^{4}-\frac{15}{4} t^{3}+\frac{18689}{120960} t-\frac{769}{181440}\right] \\
& \beta_{1}=h^{2}\left[\frac{-81}{224} t^{8}+\frac{81}{28} t^{7}-\frac{363}{40} t^{6}+\frac{279}{20} t^{5}-\frac{127}{12} t^{4}+\frac{10}{3} t^{3}-\frac{139}{864} t+\frac{1987}{136080}\right] \\
& \beta_{\frac{4}{3}}=h^{2}\left[\frac{243}{896} t^{8}-\frac{459}{224} t^{7}+\frac{963}{160} t^{6}-\frac{2763}{320} t^{5}+\frac{99}{16} t^{4}-\frac{15}{8} t^{3}+\frac{10921}{120960} t-\frac{1609}{181440}\right] \\
& \beta_{\frac{5}{3}}=h^{2}\left[\frac{-243}{2240} t^{8}+\frac{27}{35} t^{7}-\frac{171}{80} t^{6}+\frac{117}{40} t^{5}-\frac{81}{40} t^{4}+\frac{3}{5} t^{3}-\frac{347}{12096} t+\frac{263}{90720}\right] \\
& \beta_{2}=h^{2}\left[\frac{81}{4480} t^{8}-\frac{27}{224} t^{7}+\frac{51}{160} t^{6}-\frac{27}{64} t^{5}+\frac{137}{480} t^{4}-\frac{1}{12} t^{3}+\frac{479}{120960} t-\frac{221}{544320}\right]
\end{aligned}
$$

where $t=\frac{x-x_{n}}{h}$,

Evaluating (7) at non-interpolating points i.e at the points $x=x_{n+\frac{i}{3}}$ for $i=0,3,4,5,6$ which is equivalent to $t=\frac{i}{3}$

$$
\begin{aligned}
& y_{n}=\quad 2 y_{n+\frac{1}{3}}-y_{n+\frac{2}{3}}+h^{2}\left(\frac{863}{108864} f_{n}+\frac{8999}{90720} f_{n+\frac{1}{3}}-\frac{769}{181440} f_{n+\frac{2}{3}}+\frac{1987}{136080} f_{n+1}\right. \\
& \left.-\frac{1609}{181440} f_{n+\frac{4}{3}}+\frac{263}{90720} f_{n+\frac{5}{3}}-\frac{221}{544320} f_{n+2}\right)^{3} \\
& y_{n+1}=\quad-y_{n+\frac{1}{3}}+2 y_{n+\frac{2}{3}}+h^{2}\left(\frac{-221}{544320} f_{n}+\frac{977}{90720} f_{n+\frac{1}{3}}+\frac{16451}{181440} f_{n+\frac{2}{3}}+\frac{1357}{136080} f_{n+1}\right. \\
& \left.+\frac{71}{181440} f_{n+\frac{4}{3}}-\frac{31}{90720} f_{n+\frac{5}{3}}+\frac{31}{544320} f_{n+2}\right) \\
& y_{n+\frac{4}{3}}=-2 y_{n+\frac{1}{3}}+3 y_{n+\frac{2}{3}}+h^{2}\left(\frac{-137}{181440} f_{n}+\frac{209}{10080} f_{n+\frac{1}{3}}+\frac{433}{2240} f_{n+\frac{2}{3}}+\frac{4927}{45360} f_{n+1}\right. \\
& \left.+\frac{257}{20160} f_{n+\frac{4}{3}}-\frac{1}{672} f_{n+\frac{5}{3}}+\frac{31}{181440} f_{n+2}\right) \\
& y_{n+\frac{5}{3}}=-3 y_{n+\frac{1}{3}}+4 y_{n+\frac{2}{3}}+h^{2}\left(\frac{-19}{181440} f_{n}+\frac{17}{560} f_{n+\frac{1}{3}}+\frac{2987}{10080} f_{n+\frac{2}{3}}+\frac{4927}{22680} f_{n+1}\right. \\
& \left.+\frac{389}{3360} f_{n+\frac{4}{3}}+\frac{41}{5040} f_{n+\frac{5}{3}}-\frac{11}{90720} f_{n+2}\right) \\
& y_{n+2}=-4 y_{n+\frac{1}{3}}+5 y_{n+\frac{2}{3}}+h^{2}\left(\frac{-95}{54432} f_{n}+\frac{389}{9072} f_{n+\frac{1}{3}}+\frac{7085}{18144} f_{n+\frac{2}{3}}+\frac{4633}{13608} f_{n+1}\right. \\
& \left.-\frac{3893}{18144} f_{n+\frac{4}{3}}+\frac{1061}{9072} f_{n+\frac{5}{3}}+\frac{409}{54432} f_{n+2}\right)
\end{aligned}
$$


Differentiating $\alpha_{\frac{1}{3}}, \alpha_{\frac{2}{3}}$ and all the $\beta_{\frac{i}{3}}, i=0(1) 6$ and evaluating the derivative of (8) at the points $x=x_{\mathrm{n}+\frac{i}{3}}, i=0(1) 6$, equivalent to $t=\frac{i}{3}$.

$$
\begin{aligned}
h y_{n}^{\prime}+3 y_{n+\frac{1}{3}}-3 y_{n+\frac{2}{3}}=\quad & h^{2}\left[\frac{-459}{4480} f_{n}-\frac{27623}{60480} f_{n+\frac{1}{3}}+\frac{18689}{120960} f_{n+\frac{2}{3}}-\frac{139}{864} f_{n+1}+\frac{10921}{120960} f_{n+\frac{4}{3}}\right. \\
& \left.-\frac{347}{12096} f_{n+\frac{5}{3}}+\frac{479}{120960} f_{n+2}\right] \\
h y_{n+\frac{1}{3}}^{\prime}+3 y_{n+\frac{1}{3}}-3 y_{n+\frac{2}{3}}= & h^{2}\left[\frac{199}{72576} f_{n}-\frac{1973}{20160} f_{n+\frac{1}{3}}-\frac{18}{128} f_{n+\frac{2}{3}}+\frac{4157}{90720} f_{n+1}-\frac{851}{40320} f_{n+\frac{4}{3}}\right. \\
& \left.+\frac{41}{6720} f_{n+\frac{5}{3}}+\frac{289}{120960} f_{n+2}\right] \\
y_{n+\frac{2}{3}}^{\prime}+3 y_{n+\frac{1}{3}}-3 y_{n+\frac{2}{3}}= & h^{2}\left[\frac{-731}{362880} f_{n}-\frac{13}{320} f_{n+\frac{1}{3}}+\frac{6347}{40320} f_{n+\frac{2}{3}}-\frac{3971}{90720} f_{n+1}+\frac{257}{13440} f_{n+\frac{4}{3}}\right. \\
& \left.-\frac{109}{20160} f_{n+\frac{5}{3}}+\frac{253}{262880} f_{n+2}\right] \\
h y_{n+1}^{\prime}+3 y_{n+\frac{1}{3}}-3 y_{n+\frac{2}{3}}= & h^{2}\left[\frac{-1}{1920} f_{n}+\frac{1537}{60480} f_{n+\frac{1}{3}}+\frac{39587}{120960} f_{n+\frac{3}{3}}+\frac{4927}{30240} f_{n+1}-\frac{2201}{120960} f_{n+\frac{4}{3}}\right. \\
& \left.+\frac{209}{6720} f_{n+\frac{5}{3}}-\frac{43}{120960} f_{n+2}\right] \\
h y_{n+\frac{4}{3}}^{\prime}+3 y_{n+\frac{1}{3}}-3 y_{n+\frac{2}{3}=}= & h^{2}\left[\frac{-571}{362880} f_{n}+\frac{691}{20160} f_{n+\frac{1}{3}}+\frac{1299}{4480} f_{n+\frac{3}{3}}+\frac{33533}{90720} f_{n+1}+\frac{1223}{8064} f_{n+\frac{4}{3}}\right. \\
& \left.+\frac{79}{6720} f_{n+\frac{5}{3}}+\frac{59}{51840} f_{n+2}\right] \\
h y_{n+\frac{5}{3}}^{\prime}+3 y_{n+\frac{1}{3}}-3 y_{n+\frac{2}{3}=}= & h^{2}\left[\frac{-29}{362880} f_{n}+\frac{51}{2240} f_{n+\frac{1}{3}}+\frac{13313}{40320} f_{n+\frac{2}{3}}+\frac{5081}{18144} f_{n+1}-\frac{5519}{13440} f_{n+\frac{4}{3}}\right. \\
& \left.+\frac{73}{576} f_{n+\frac{5}{3}}+\frac{1313}{362880} f_{n+2}\right]
\end{aligned}
$$

The schemes in (9) and (10) form the requited method for solving (1) numerically.

\subsubsection{Case 2}

Here, we consider the specification where the off-set points are also $x_{\frac{i}{3}}$, for $i=1,2,4,5$. Interpolating (2) at the points $x_{i+n}$, for

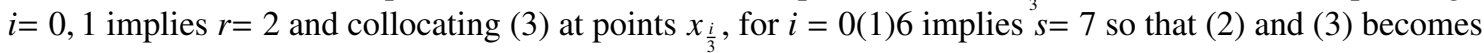

$$
y(x) \simeq p(x)=\sum_{i=0}^{8} a_{i} x^{i}=a_{0}+a_{1} x+a_{2} x^{2}+\cdots+a_{8} x^{8}
$$

which on differentiating yields

$$
y^{\prime \prime}(x) \simeq p^{\prime \prime}(x)=\sum_{i=2}^{8} i(i-1) a_{i} x^{i-2}=2 a_{2}+6 a_{3} x+12 a_{4} x^{2}+\cdots+56 a_{8} x^{6}
$$

From the imposed collocation condition, the following system of algebraic equation is obtained

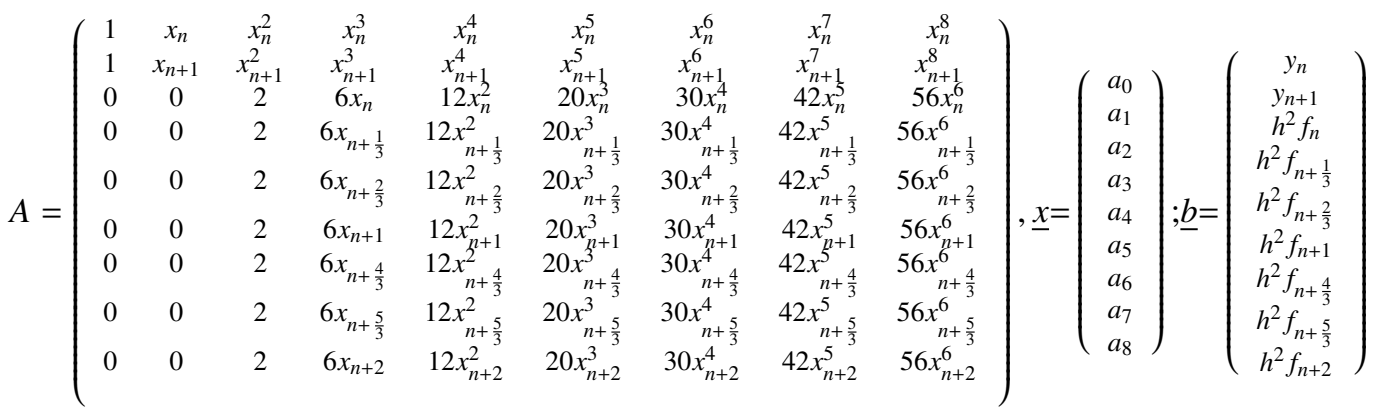

$$
\begin{aligned}
& \underline{e}=\left(1, x, x^{2}, x^{3}, x^{4}, x^{5}, x^{6}, x^{7}, x^{8}\right)^{T}
\end{aligned}
$$

Applying the above theorem, the following continuous hybrid method is derived

$$
y(x)=\sum_{i=0}^{1} \alpha_{i} y_{n+i}+h^{2} \sum_{i=0}^{2} \beta_{\frac{i}{3}} f_{n+\frac{i}{3}}
$$


where $\alpha$ and $\beta$ are function of $t$ given as

$$
\begin{aligned}
& \alpha_{0}=-t \\
& \alpha_{1}=1+t \\
& \beta_{0}=\frac{105 h^{2} t-112 h^{2} t^{3}+56 h^{2} t^{4}+378 h^{2} t^{5}-252 h^{2} t^{6}-324 h^{2} t^{7}+243 h^{2} t^{8}}{13440} \\
& \beta_{\frac{1}{3}}=-\frac{3\left(-85 h^{2} t-56 h^{2} t^{3}+42 h^{2} t^{4}+168 h^{2} t^{5}-168 h^{2} t^{6}-72 h^{2} t^{7}+81 h^{2} t^{8}\right)}{2240} \\
& \beta_{\frac{2}{3}}=\frac{3\left(347 h^{2} t-560 h^{2} t^{3}+840 h^{2} t^{4}+546 h^{2} t^{5}-1092 h^{2} t^{6}-180 h^{2} t^{7}+405 h^{2} t^{8}\right)}{4480} \\
& \beta_{1}=\frac{563 h^{2} t+1680 h^{2} t^{2}-3430 h^{2} t^{4}+3528 h^{2} t^{6}-1215 h^{2} t^{8}}{360} \\
& \beta_{\frac{4}{3}}=\frac{3\left(-41 h^{2} t+560 h^{2} t^{3}+840 h^{2} t^{4}-546 h^{2} t^{5}-1092 h^{2} t^{6}+180 h^{2} t^{7}+405 h^{2} t^{8}\right)}{4480} \\
& \beta_{\frac{5}{3}}=-\frac{3\left(-5 h^{2} t+56 h^{2} t^{3}+42 h^{2} t^{4}-168 h^{2} t^{5}-168 h^{2} t^{6}+72 h^{2} t^{7}+81 h^{2} t^{8}\right)}{2240} \\
& \beta_{2}=\frac{-11 h^{2} t+112 h^{2} t^{3}+56 h^{2} t^{4}-378 h^{2} t^{5}-252 h^{2} t^{6}+324 h^{2} t^{7}+243 h^{2} t^{8}}{13440}
\end{aligned}
$$

where $t=\frac{x-x_{n-1}}{h}$.

Evaluating (13) at the points $x=x_{n+\frac{i}{3}}$ for $i=1,2,4,5,6$ which is equivalent to $t=-2 / 3,-1 / 3,1 / 3,2 / 3,1$ the following main methods are obtained

$$
\begin{aligned}
& y_{n+\frac{1}{3}}=\frac{2 y_{n}}{3}+\frac{y_{n+1}}{3}-h^{2}\left(\frac{2803 f_{n}}{544320}-\frac{1265 f_{n+\frac{1}{3}}}{18144}-\frac{1657 f_{n+\frac{2}{3}}}{60480}-\frac{1777 f_{n+1}}{136080}+\frac{1049 f_{n+\frac{4}{3}}}{181440}-\frac{11 f_{n+\frac{5}{3}}}{6048}+\frac{137 f_{n+2}}{544320}\right) \\
& y_{n+\frac{2}{3}}=\frac{y_{n}}{3}+\frac{2 y_{n+1}}{3}-h^{2}\left(\frac{1291 f_{n}}{544320}-\frac{1217 f_{n+\frac{1}{3}}}{30240}-\frac{10711 f_{n+\frac{2}{3}}}{181440}-\frac{1567 f_{n+1}}{136080}+\frac{163 f_{n+\frac{4}{3}}}{60480}-\frac{67 f_{n+\frac{5}{3}}}{90720}+\frac{53 f_{n+2}}{544320}\right) \\
& y_{n+\frac{4}{3}}=-\frac{y_{n}}{3}+\frac{4 y_{n+1}}{3}+h^{2}\left(\frac{661 f_{n}}{272160}+\frac{1789 f_{n+\frac{1}{3}}}{45360}+\frac{2147 f_{n+\frac{2}{3}}}{30240}+\frac{6817 f_{n+1}}{68040}+\frac{841 f_{n+\frac{4}{3}}}{90720}-\frac{f_{n+\frac{5}{3}}}{15120}-\frac{11 f_{n+2}}{272160}\right) \\
& y_{n+\frac{5}{3}}=-\frac{2 y_{n}}{3}+\frac{5 y_{n+1}}{3}+h^{2}\left(\frac{535 f_{n}}{108864}+\frac{475 f_{n+\frac{1}{3}}}{6048}+\frac{5167 f_{n+\frac{2}{3}}}{36288}+\frac{5725 f_{n+1}}{27216}+\frac{1321 f_{n+\frac{4}{3}}}{12096}+\frac{193 f_{n+\frac{5}{3}}}{18144}-\frac{53 f_{n+2}}{108864}\right) \\
& y_{n+2}=-y_{n}+2 y_{n+1}+h^{2}\left(\frac{47 f_{n}}{6720}+\frac{27}{224} f_{n+\frac{1}{3}}+\frac{459 f_{n+\frac{2}{3}}}{2240}+\frac{563 f_{n+1}}{1680}+\frac{459 f_{n+\frac{4}{3}}}{2240}+\frac{27}{224} f_{n+\frac{5}{3}}+\frac{47 f_{n+2}}{6720}\right)
\end{aligned}
$$

differentiating (14) and evaluating the derivative of (13) at the points $x=x_{n+\frac{i}{3}}$ for $i=0(1) 6$ which is equivalent to $t=-1,-2 / 3,-1 / 3,0,1 / 3,2 / 3,1$ the following additional methods are obtained

$$
\begin{aligned}
& y_{n}^{\prime}=-\frac{y_{n+n}}{h}+h\left(\frac{253 f_{n}}{2688}-\frac{165}{448} f_{n+\frac{1}{3}}+\frac{267 f_{n+\frac{2}{3}}}{4480}-\frac{5}{32} f_{n+1}+\frac{363 f_{n+\frac{4}{3}}}{4480}-\frac{57 f_{n+\frac{5}{3}}}{2240}+\frac{47 f_{n+2}}{13440}\right), \\
& y^{\prime}{ }_{n+\frac{1}{3}}=-\frac{y_{n}}{h}+\frac{y_{n+1}}{h}+h\left(\frac{4019 h f_{n}}{362880}-\frac{571 h f_{n+\frac{1}{3}}}{60480}-\frac{679 h f_{n+\frac{2}{3}}}{3456}+\frac{4577 h f_{n+1}}{90720}-\frac{3673 h f_{n+\frac{4}{3}}}{120960}+\frac{113 h f_{n+\frac{5}{3}}}{12096}-\frac{457 h f_{n+2}}{362880}\right), \\
& y_{n+\frac{2}{3}}^{\prime}=-\frac{y_{n}}{h}+\frac{y_{n+1}}{h}+h\left(\frac{2293 h f_{n}}{362880}+\frac{223 h f_{n+\frac{1}{3}}}{1728}+\frac{7561 h f_{n+\frac{2}{3}}}{120960}-\frac{3551 h f_{n+1}}{90720}+\frac{1193 h f_{n+\frac{4}{3}}}{120960}-\frac{131 h f_{n+\frac{5}{3}}}{60480}+\frac{17 h f_{n+2}}{72576}\right), \\
& y_{n+1}^{\prime}=-\frac{y_{n}}{h}+\frac{y_{n+1}}{h}+h\left(\frac{h f_{n}}{128}+\frac{51}{448} h f_{n+\frac{1}{3}}+\frac{1041 h f_{n+\frac{2}{3}}}{4480}+\frac{563 h f_{n+1}}{3360}-\frac{123 h f_{n+\frac{4}{3}}}{4480}+\frac{3}{448} h f_{n+\frac{5}{3}}-\frac{11 h f_{n+2}}{13440}\right), \\
& y_{n+\frac{4}{3}}^{\prime}=-\frac{y_{n}}{h}+\frac{y_{n+1}}{h}+h\left(\frac{2453 h f_{n}}{362880}+\frac{7421 h f_{n+\frac{1}{3}}}{60480}+\frac{23593 h f_{n+\frac{2}{3}}}{120960}+\frac{33953 h f_{n+1}}{90720}+\frac{3445 h f_{n+\frac{4}{3}}}{24192}-\frac{103 h f_{n+\frac{5}{3}}}{12096}+\frac{7 h f_{n+2}}{10368}\right), \\
& y_{n+\frac{5}{3}}^{\prime}=-\frac{y_{n}}{h}+\frac{y_{n+1}}{h}+h\left(\frac{599 h f_{n}}{72576}+\frac{1345 h f_{n+\frac{1}{3}}}{12096}+\frac{28459 h f_{n+\frac{2}{3}}}{120960}+\frac{5165 h f_{n+1}}{18144}+\frac{48551 h f_{n+\frac{4}{3}}}{120960}+\frac{1123 h f_{n+\frac{5}{3}}}{8640}-\frac{1481 h f_{n+2}}{362880}\right), \\
& y^{\prime}{ }_{n+2}=-\frac{y_{n}}{h}+\frac{y_{n+1}}{h}+h\left(\frac{47 h f_{n}}{13440}+\frac{327 h f_{n+\frac{1}{3}}}{2240}+\frac{111}{896} h f_{n+\frac{2}{3}}+\frac{1651 h f_{n+1}}{3360}+\frac{93}{640} h f_{n+\frac{4}{3}}+\frac{219}{448} h f_{n+\frac{5}{3}}+\frac{453 h f_{n+2}}{4480}\right)
\end{aligned}
$$

Here, it is noteworthy that (9) and (10) are combined to form a block for case 1 while (15) and (16) form another block for case 11. For each case, (1) is solved which is a system of second-order ordinary differential equations resulting from the semidiscretization of a second-order PDE.

\section{Analysis of the Method}

\subsection{Order and Local Truncation Error (LTE)}

The LMMs (8), and (13) are said to be of order $p$ if

$$
C_{0}=C_{1}=C_{2}=\cdots=C_{p}+\mu-1=0, \quad C_{p+\mu} \neq 0 .
$$


Here $C_{p+\mu}$ is the error constant and

$$
C_{p+\mu} h^{p+\mu} y^{(p+\mu)}\left(x_{n}\right)
$$

is the principal Local Truncation Error (LTE) at the point $x_{n}$. The $C^{\prime} s$ are given by

$$
\begin{aligned}
& C_{0}=\alpha_{0}+\alpha_{1}+\alpha_{2}+\cdots+\alpha_{k} \\
& C_{1}=\left(\alpha_{0}+\alpha_{1}+\alpha_{2}+\cdots+\alpha_{k}\right)-\left(\beta_{0}+\beta_{1}+\cdots+\beta_{k}\right) \\
& C_{q}=\frac{1}{q !}\left(\alpha_{1}+2^{q} \alpha_{2}+\cdots+k^{q} \alpha_{k}\right)-\frac{1}{(q-3) !}\left(\beta_{1}+2^{q-1} \beta_{2}+\cdots+k^{q-3} \beta_{k}\right), q=2,3, \ldots
\end{aligned}
$$

The LTE associated with any of (8) and (13) is given by the difference operator

$$
L[y(x): h]=\sum_{i=1}^{2} \alpha_{\frac{i}{3}} y\left(x_{n}+\frac{i}{3}\right)-h^{2} \sum_{j=0}^{2} \beta_{\frac{i}{3}} y^{\prime \prime}\left(x_{n}+\frac{i}{3}\right)
$$

where $y \in C^{2}[a, b]$ is an arbitrary function. Expanding (17) in Taylor's series about the point $x_{n}$, the expression is obtained as:

$$
L\left[y\left(x_{n}\right): h\right]=C_{0} y\left(x_{n}\right)+C_{1} h y^{\prime}\left(x_{n}\right)+C_{2} h^{2} y^{\prime \prime}\left(x_{n}\right)+\cdots+C_{\rho+2} h^{\rho+2} y^{\rho+2}\left(x_{n}\right)
$$

Expanding each scheme in (9) and (10), the following principal truncation errors are obtained:

$$
\begin{aligned}
& C_{p+2}^{0}=-\frac{19 y^{(9)}[x] h^{9}}{119042784}+O[h]^{10}, C_{p+2}^{1}=\frac{31 y^{(9)}[x] h^{9}}{1190427840}+O[h]^{10}, C_{p+2}^{2}=\frac{y^{(9)}[x] h^{9}}{4723920}+O[h]^{10} \\
& C_{p+2}^{\frac{4}{3}}=\frac{31 y^{(9)}[x] h^{9}}{595213920}+O[h]^{10}, C_{p+2}^{\frac{5}{3}}=\frac{31 y^{(9)}[x] h^{9}}{595213920}+O[h]^{10}, C^{\prime 0}{ }_{p+2}=\frac{8881 y^{(9)}[x] h^{9}}{5952139200}+O[h]^{10} \\
& C_{p+2}^{\prime \frac{1}{3}}=-\frac{409 y^{(9)}[x] h^{9}}{1700611200}+O[h]^{10}, C^{\prime \frac{2}{3}}{ }_{p+2}=\frac{1201 y^{(9)}[x] h^{9}}{5952139200}+O[h]^{10}, C_{p+2}{ }_{p+2}=-\frac{463 y^{(9)}[x] h^{9}}{11904278400}+O[h]^{10} \\
& C^{\prime \frac{4}{3}}{ }_{p+2}=\frac{1201 y^{(9)}[x] h^{9}}{5952139200}+O[h]^{10}, C^{\prime \frac{5}{3}}{ }_{p+2}=-\frac{409 y^{(9)}[x] h^{9}}{1700611200}+O[h]^{10}, C^{\prime 2}{ }_{p+2}=\frac{8881 y^{(9)}[x] h^{9}}{5952139200}+O[h]^{10}
\end{aligned}
$$

The above blocked method (9) and (10) is of uniform order $p=7$

Expanding each scheme in (15) and (16), the following principal truncation errors are obtained:

$$
\begin{aligned}
& C_{p+2}^{\frac{1}{3}}=\frac{349 y^{(9)}(x) h^{9}}{3571283520}+O(h)^{10}, C_{p+2}^{\frac{2}{3}}=\frac{2 y^{(9)}(x) h^{9}}{55801305}+O(h)^{10}, C_{p+2}^{\frac{4}{3}}=-\frac{2 y^{(9)}(x) h^{9}}{55801305}+O(h)^{10} \\
& C_{p+2}^{\frac{5}{3}}=-\frac{349 y^{(9)}(x) h^{9}}{3571283520}+O(h)^{10}, C_{p+2}^{2}=\frac{y^{(9)}(x) h^{9}}{32659200}+O(h)^{10}, C_{p+2}^{\prime 0}=\frac{y^{(9)}(x) h^{9}}{765450}+O(h)^{10} \\
& C_{p+2}^{\prime \frac{1}{3}}=-\frac{1691 y^{(9)}(x) h^{9}}{3968092800}+O(h)^{10}, C_{p+2}^{\prime \frac{2}{3}}=\frac{y^{(9)}(x) h^{9}}{62001450}+O(h)^{10}, C_{p+2}^{\prime 1}-\frac{11 y^{(9)}(x) h^{9}}{48988800}+O(h)^{10} \\
& C_{p+2}^{\prime \frac{4}{3}} \frac{y^{(9)}(x) h^{9}}{62001450}+O(h)^{10}, C_{p+2}^{\prime \frac{5}{3}}-\frac{1691 y^{(9)}(x) h^{9}}{3968092800}+O(h)^{10}, C_{p+2}^{\prime 2} \frac{y^{(9)}(x) h^{9}}{765450}++O(h)^{10}
\end{aligned}
$$

The above blocked method (15) and (16) is of uniform order $p=7$

The LMM (8) (same for (13)) is said to be consistent if it has order $p \geq 1$ and the first and second characteristic polynomials which are defined respectively, as

$$
\rho(r)=\sum_{j=0}^{k} \alpha_{j} z^{j}
$$

and

$$
\sigma(r)=\sum_{j=0}^{k} \beta_{j} z^{j}
$$


where $r$ is the principal root, satisfy the following conditions:

$$
\begin{aligned}
& \sum_{j=0}^{k} \alpha_{j}=0 \\
& \rho(1)=\rho^{\prime}(1)=0
\end{aligned}
$$

and

$$
\rho^{\prime \prime}(1)=2 ! \sigma(1)
$$

Henrici [15], Lambert[16].

Consider the main method in (9) given as

$$
\begin{aligned}
y_{n+2}= & -4 y_{n+\frac{1}{3}}+5 y_{n+\frac{2}{3}}+h^{2}\left(\frac{-95}{54432} f_{n}+\frac{389}{9072} f_{n+\frac{1}{3}}+\frac{7085}{18144} f_{n+\frac{2}{3}}+\frac{4633}{13608} f_{n+1}\right. \\
& \left.-\frac{3893}{18144} f_{n+\frac{4}{3}}+\frac{1061}{9072} f_{n+\frac{5}{3}}+\frac{409}{54432} f_{n+2}\right)
\end{aligned}
$$

The condition (21) is satisfied. the first characteristic equation for (9) is given as:

$$
\begin{aligned}
& \rho(r)=r^{2}+4 r^{\frac{1}{3}}-5 r^{\frac{2}{3}} \\
& \rho^{\prime}(r)=2 r+\frac{4}{3 r^{2 / 3}}-\frac{10}{3 r^{1 / 3}}
\end{aligned}
$$

Here $\rho(r)=0, \rho^{\prime}(r)=0$. Therefore, (22) is satisfied. The second characteristic polynomial for (9) is given as

$$
\begin{aligned}
& \sigma(r)=\frac{-95}{54432}+\frac{389}{9072} r^{\frac{1}{3}}+\frac{7085}{18144} r^{\frac{2}{3}}+\frac{4633}{13608} r+\frac{3893}{18144} r^{\frac{4}{3}}+\frac{1061}{9072} r^{\frac{5}{3}}+\frac{409}{54432} r^{2} \\
& \sigma(1)=\frac{10}{9} \\
& \rho^{\prime \prime}(r)=2-\frac{8}{9 r^{5 / 3}}+\frac{10}{9 r^{4 / 3}} . \quad \rho^{\prime \prime}(r)=\frac{20}{9}
\end{aligned}
$$

Hence condition (23) is satisfied. Conclusively, the hybrid method is consistent.

Consider the main method in (15) given as

$$
\begin{aligned}
y_{n+2}= & -y_{n}+2 y_{n+1}+h^{2}\left(\frac{47 f_{n}}{6720}+\frac{27}{224} f_{n+\frac{1}{3}}+\frac{459 f_{n+\frac{2}{3}}}{2240}+\frac{563 f_{n+1}}{1680}\right. \\
& \left.+\frac{459 f_{n+\frac{4}{3}}}{2240}+\frac{27}{224} f_{n+\frac{5}{3}}+\frac{47 f_{n+2}}{6720}\right)
\end{aligned}
$$

The condition (21) is satisfied. the first characteristic equation for (15) is given as:

$$
\begin{aligned}
& \rho(r)=r^{2}-2 r+1 \\
& \rho^{\prime}(r)=2 r-2
\end{aligned}
$$

Here $\rho(1)=0, \rho^{\prime}(1)=0$. Therefore, (22) is satisfied. The second characteristic polynomial for (15) is given as

$$
\begin{aligned}
& \sigma(r)=\frac{47}{6720}+\frac{27}{224} r^{\frac{1}{3}}+\frac{459}{2240} r^{\frac{2}{3}}+\frac{563}{1680} r+\frac{459}{2240} r^{\frac{4}{3}}+\frac{27}{224} r^{\frac{5}{3}}+\frac{47}{6720} r^{2} \\
& \sigma(1)=1 \\
& \rho^{\prime \prime}(r)=2 . \quad \rho^{\prime \prime}(1)=2
\end{aligned}
$$

Hence condition (23) is satisfied. Conclusively, the hybrid method is consistent. 


\subsection{Zero Stability}

To establish hat the methods are zero stable, each of the method in block form are solved simultaneously to obtain all the $y_{i}$ and $y_{i}^{\prime}$ 's for appropriate index $i$, (see Modebei et al.[17]). For the method (9) and its additional methods in (10), they are taken in block form and solved simultaneously to obtain $y_{\frac{i}{3}}$ for $i=1(1) 6$ to obtain the following block method. For block method (9)-(10)

$$
\begin{aligned}
& y_{n+\frac{1}{3}}=y_{n}+\frac{h y_{n}^{\prime}}{3}+h^{2}\left(\frac{28549 f_{n}}{1088640}+\frac{275 f_{n+\frac{1}{3}}}{5184}-\frac{5717 f_{n+\frac{2}{3}}}{120960}+\frac{10621 f_{n+1}}{272160}-\frac{7703 f_{n+\frac{4}{3}}}{362880}+\frac{403 f_{n+\frac{5}{3}}}{60480}-\frac{199 f_{n+2}}{217728}\right) \\
& y_{n+\frac{2}{3}}=y_{n}+\frac{2 h y^{\prime}{ }_{n}}{3}+h^{2}\left(\frac{1027 f_{n}}{17010}+\frac{194}{945} f_{n+\frac{1}{3}}-\frac{8}{81} f_{n+\frac{2}{3}}+\frac{788 f_{n+1}}{8505}-\frac{97 f_{n+\frac{4}{3}}}{1890}+\frac{46 f_{n+\frac{5}{3}}}{2835}-\frac{19 f_{n+2}}{8505}\right) \\
& y_{n+1}=\quad y_{n}+h y^{\prime}{ }_{n}+h^{2}\left(\frac{253 f_{n}}{2688}+\frac{165}{448} f_{n+\frac{1}{3}}-\frac{267 f_{n+\frac{2}{3}}}{4480}+\frac{5 f_{n+1}}{32}-\frac{363 f_{n+\frac{4}{3}}}{4480}+\frac{57 f_{n+\frac{5}{3}}}{2240}-\frac{47 f_{n+2}}{13440}\right) \\
& y_{n+\frac{4}{3}}=y_{n}+\frac{4 h y^{\prime}{ }_{n}}{3}+h^{2}\left(\frac{1088 f_{n}}{8505}+\frac{1504 f_{n+\frac{1}{3}}}{2835}-\frac{8}{945} f_{n+\frac{2}{3}}+\frac{2624 f_{n+1}}{8505}-\frac{8}{81} f_{n+\frac{4}{3}}+\frac{32}{945} f_{n+\frac{5}{3}}-\frac{8 f_{n+2}}{1701}\right) \\
& y_{n+\frac{5}{3}}=y_{n}+\frac{5 h y_{n}^{\prime}}{3}+h^{2}\left(\frac{35225 f_{n}}{217728}+\frac{8375 f_{n+\frac{1}{3}}}{12096}+\frac{3125 f_{n+\frac{2}{3}}}{72576}+\frac{25625 f_{n+1}}{54432}-\frac{625 f_{n+\frac{4}{3}}}{24192}+\frac{275 f_{n+\frac{5}{3}}}{5184}-\frac{1375 f_{n+2}}{217728}\right) \\
& y_{n+2}=y_{n}+2 h y_{n}^{\prime}+h^{2}\left(\frac{41 f_{n}}{210}+\frac{6}{7} f_{n+\frac{1}{3}}+\frac{3}{35} f_{n+\frac{2}{3}}+\frac{68 f_{n+1}}{105}+\frac{3}{70} f_{n+\frac{4}{3}}+\frac{6}{35} f_{n+\frac{5}{3}}\right) \\
& y_{n+\frac{1}{3}}^{\prime}=y_{n}^{\prime}+h\left(\frac{19087 f_{n}}{181440}+\frac{2713 f_{n+\frac{1}{3}}}{7560}-\frac{15487 f_{n+\frac{2}{3}}}{60480}+\frac{586 f_{n+1}}{2835}-\frac{6737 f_{n+\frac{4}{3}}}{60480}+\frac{263 f_{n+\frac{5}{3}}}{7560}-\frac{863 f_{n+2}}{181440}\right) \\
& y_{n+\frac{2}{3}}^{\prime}=y_{n}^{\prime}+h\left(\frac{1139 f_{n}}{11340}+\frac{94}{189} f_{n+\frac{1}{3}}+\frac{11 f_{n+\frac{2}{3}}}{3780}+\frac{332 f_{n+1}}{2835}-\frac{269 f_{n+\frac{4}{3}}}{3780}+\frac{22}{945} f_{n+\frac{5}{3}}-\frac{37 f_{n+2}}{11340}\right) \\
& y_{n+1}^{\prime}=y_{n}^{\prime}+h\left(\frac{137 f_{n}}{1344}+\frac{27}{56} f_{n+\frac{1}{3}}+\frac{387 f_{n+\frac{2}{3}}}{2240}+\frac{34 f_{n+1}}{105}-\frac{243 f_{n+\frac{4}{3}}}{2240}+\frac{9}{280} f_{n+\frac{5}{3}}-\frac{29 f_{n+2}}{6720}\right) \\
& y_{n+\frac{4}{3}}^{\prime}=y_{n}^{\prime}+h\left(\frac{286 f_{n}}{2835}+\frac{464}{945} f_{n+\frac{1}{3}}+\frac{128}{945} f_{n+\frac{2}{3}}+\frac{1504 f_{n+1}}{2835}+\frac{58}{945} f_{n+\frac{4}{3}}+\frac{16}{945} f_{n+\frac{5}{3}}-\frac{8 f_{n+2}}{2835}\right) \\
& y_{n+\frac{5}{3}}^{\prime}=y_{n}^{\prime}+h\left(\frac{3715 f_{n}}{36288}+\frac{725 f_{n+\frac{1}{3}}}{1512}+\frac{2125 f_{n+\frac{2}{3}}}{12096}+\frac{250 f_{n+1}}{567}+\frac{3875 f_{n+\frac{4}{3}}}{12096}+\frac{235 f_{n+\frac{5}{3}}}{1512}-\frac{275 f_{n+2}}{36288}\right) \\
& y_{n+2}^{\prime}=y_{n}^{\prime}+h\left(\frac{41 f_{n}}{420}+\frac{18}{35} f_{n+\frac{1}{3}}+\frac{9}{140} f_{n+\frac{2}{3}}+\frac{68 f_{n+1}}{105}+\frac{9}{140} f_{n+\frac{4}{3}}+\frac{18}{35} f_{n+\frac{5}{3}}+\frac{41 f_{n+2}}{420}\right)
\end{aligned}
$$

For block method (15)-(16) similar operation is carried out;

A numerical method is zero-stable if the solutions remain bounded as $h \rightarrow 0$, which means that the method does not provide solutions that grow unbounded as the number of steps increases, Modebei et al.[17]. To show the zero-stability of the block method (36), we take $h \rightarrow 0$ the method may be rewritten in matrix form as

$$
\begin{aligned}
& A_{0} Y_{n}=A_{1} Y_{n-1} \\
& Y_{n}=\left(Y_{n}^{0}, Y_{n}^{1}\right)^{T} \\
& Y_{n}^{0}=\left(y_{n+\frac{1}{3}}, y_{n+\frac{2}{3}}, y_{n+1}, y_{n+\frac{4}{3}}, y_{n+\frac{5}{3}}, y_{n+2}\right)^{T} \\
& Y_{n}^{1}=\left(y^{\prime}{ }_{n+\frac{1}{3}}, y_{n+\frac{2}{3}}^{\prime}, y_{n+1}^{\prime}, y_{n+\frac{4}{3}}^{\prime}, y_{n+\frac{5}{3}}^{\prime}, y_{n+2}^{\prime}\right)^{T}
\end{aligned}
$$

For method (36) $A_{0}=I_{12 \times 12}$ identity matrix and $A_{1}=I_{12 \times 12}$ matrix given by

$$
A_{1}=\left(\begin{array}{ll}
A_{11} & 0 \\
0 & A_{22} \\
&
\end{array}\right), A_{11}=\left(\begin{array}{cccccc}
1 & 0 & 0 & 0 & 0 & 0 \\
1 & 0 & 0 & 0 & 0 & 0 \\
1 & 0 & 0 & 0 & 0 & 0 \\
1 & 0 & 0 & 0 & 0 & 0 \\
1 & 0 & 0 & 0 & 0 & 0 \\
1 & 0 & 0 & 0 & 0 & 0
\end{array}\right), A_{22}=\left(\begin{array}{cccccc}
0 & 1 & 0 & 0 & 0 & 0 \\
0 & 1 & 0 & 0 & 0 & 0 \\
0 & 1 & 0 & 0 & 0 & 0 \\
0 & 1 & 0 & 0 & 0 & 0 \\
0 & 1 & 0 & 0 & 0 & 0 \\
0 & 1 & 0 & 0 & 0 & 0
\end{array}\right)
$$


The characteristic polynomial of the matrix $A_{11}$ is given as $\left|A_{11}-\lambda I\right|$, that is $\lambda^{5}(\lambda-1)=0$ with root $\lambda_{j}=0$ for $j=1, \ldots, 5$ and $\lambda_{6}=1$.

The characteristic polynomial of the matrix $A_{22}$ is given as $\left|A_{22}-\lambda I\right|$, that is $\lambda^{5}(\lambda-1)=0$ with $\operatorname{root} \lambda_{j}=0$ for $j=1, \ldots, 5$ and $\lambda_{6}=1$.

For method (15)-(16) $A_{0}=I_{12 \times 12}$ identity matrix and $A_{1}=I_{12 \times 12}$ matrix given by

$$
A_{1}=\left(\begin{array}{ll}
A_{11} & 0 \\
0 & A_{22} \\
&
\end{array}\right), A_{11}=A_{22}=\left(\begin{array}{cccccc}
1 & 0 & 0 & 0 & 0 & 0 \\
1 & 0 & 0 & 0 & 0 & 0 \\
1 & 0 & 0 & 0 & 0 & 0 \\
1 & 0 & 0 & 0 & 0 & 0 \\
1 & 0 & 0 & 0 & 0 & 0 \\
1 & 0 & 0 & 0 & 0 & 0
\end{array}\right)
$$

The characteristic polynomial of the matrix $A_{i i}$ is given as $\left|A_{i i}-\lambda I\right|$, $i=1,2$, that is $\lambda^{5}(\lambda-1)=0$ with root $\lambda_{j}=0$ for $j=1, \ldots, 5$ and $\lambda_{6}=1$.

Definition 3.1. The two step hybrid block method (9)-(10) (or (15)-(16)) is said to be zero stabile if the number of root of the first characteristic equation $|\rho(r)|<1$ and if $|\rho(r)|=1$, then the multiplicity of $\rho(r)$ must not exceed 2 . Hence, the are zero stable.

\subsection{Convergence of the Methods}

Definition 3.2. Convergence: An LMM is said to be convergent if and only if it is consistent and zero-stable.

By the above definition, the derived hybrid methods are convergent.

\section{Numerical Examples}

In this section, the performance of the developed two-step hybrid block scheme is examined. the exact and approximate solution are tabulated. The tables below show the numerical results of the newly developed scheme with the exact solution for solving the problem and the result of the developed scheme are more accurate than existing methods. For simplicity, method in (9)-(10) would be termed Hybrid 2-step Block Method 1 (H2BM1), and method in (15)-(16) would be termed Hybrid 2-step Block Method 2 (H2BM2)

\section{Example 1.}

Consider the PDE (Ngwane and Jator [10]).

$$
\begin{aligned}
& \kappa \frac{\partial y^{2}}{\partial x^{2}}-\frac{\partial y}{\partial t}=0 \\
& y(0, t)=y(1, t)=0, \quad y(x, 0)=\sin \pi x+\sin \omega \pi x, \quad \kappa>1
\end{aligned}
$$

The analytic solution is $y(x, t)=e^{-\pi^{2} \kappa t} \sin \pi x+e^{-\omega^{2} \pi^{2} \kappa t} \sin \pi x$ Following Ngwane and Jator [10], (38) becomes

$$
\begin{aligned}
& \frac{\mathrm{d} y_{m}^{2}}{\mathrm{~d} x^{2}}=\frac{1}{\kappa} \frac{\left.y\left(x, t_{m+1}\right)-y\left(x, t_{m-1}\right)\right]}{(\Delta t)} \\
& y_{m}\left(0, t_{m}\right)=y_{m}\left(1, t_{m}\right)=0, \quad y_{m}(x, 0)=\sin \pi x+\sin \omega \pi x, \quad \kappa>1
\end{aligned}
$$

Table 1: Exact and Numerical solution for Example 1

\begin{tabular}{cccc}
\hline $\mathrm{x}$ & Exact & H2BM1 & Error \\
\hline 0.0 & $1.65341 \mathrm{E}-9$ & $1.65341 \mathrm{E}-9$ & 0 \\
0.1 & $6.16242 \mathrm{E}-10$ & $6.16242 \mathrm{E}-10$ & $2.78 \mathrm{E}-12$ \\
0.2 & $2.29678 \mathrm{E}-10$ & $2.29678 \mathrm{E}-10$ & $4.45 \mathrm{E}-12$ \\
0.3 & $8.56029 \mathrm{E}-11$ & $8.56029 \mathrm{E}-11$ & $4.20 \mathrm{E}-12$ \\
0.4 & $3.19048 \mathrm{E}-11$ & $3.19048 \mathrm{E}-11$ & $5.00 \mathrm{E}-12$ \\
0.5 & $1.18911 \mathrm{E}-11$ & $1.18911 \mathrm{E}-11$ & $6.45 \mathrm{E}-12$ \\
0.6 & $4.43194 \mathrm{E}-12$ & $4.43194 \mathrm{E}-12$ & $7.31 \mathrm{E}-12$ \\
0.7 & $1.65181 \mathrm{E}-12$ & $1.65181 \mathrm{E}-12$ & $3.28 \mathrm{E}-12$ \\
0.8 & $6.15646 \mathrm{E}-13$ & $6.15646 \mathrm{E}-13$ & $4.11 \mathrm{E}-13$ \\
0.9 & $2.29456 \mathrm{E}-13$ & $2.29456 \mathrm{E}-13$ & $5.99 \mathrm{E}-13$ \\
\hline
\end{tabular}

Table 2: Exact and Numerical solution for Example 1

\begin{tabular}{cccc}
\hline 9 & Exact & H2BM2 & Error \\
\hline 0.0 & $1.65341 \mathrm{E}-9$ & $1.65341 \mathrm{E}-9$ & 0 \\
\hline 0.1 & $6.16242 \mathrm{E}-10$ & $6.16242 \mathrm{E}-10$ & $3.24 \mathrm{E}-12$ \\
0.2 & $2.29678 \mathrm{E}-10$ & $2.29678 \mathrm{E}-10$ & $7.21 \mathrm{E}-12$ \\
0.3 & $8.56029 \mathrm{E}-11$ & $8.56029 \mathrm{E}-11$ & $2.22 \mathrm{E}-12$ \\
0.4 & $3.19048 \mathrm{E}-11$ & $3.19048 \mathrm{E}-11$ & $8.45 \mathrm{E}-12$ \\
0.5 & $1.18911 \mathrm{E}-11$ & $1.18911 \mathrm{E}-11$ & $1.15 \mathrm{E}-12$ \\
0.6 & $4.43194 \mathrm{E}-12$ & $4.43194 \mathrm{E}-12$ & $1.18 \mathrm{E}-12$ \\
0.7 & $1.65181 \mathrm{E}-12$ & $1.65181 \mathrm{E}-12$ & $5.08 \mathrm{E}-12$ \\
0.8 & $6.15646 \mathrm{E}-13$ & $6.15646 \mathrm{E}-13$ & $2.48 \mathrm{E}-13$ \\
0.9 & $2.29456 \mathrm{E}-13$ & $2.29456 \mathrm{E}-13$ & $4.79 \mathrm{E}-13$ \\
\hline
\end{tabular}

where

$$
t_{m}=m \Delta t, \quad m=0,1, \ldots, ; M
$$

$$
y_{m}(x) \approx y\left(x, t_{m}\right), y(x)=\left[y_{0}(x), y_{1}(x), \ldots, \mathrm{y}_{M-1}(x)\right]^{T},
$$

hence (39) becomes the system $\frac{d^{2} y_{m}(x)}{d x^{2}}=f\left(x, y_{m}\right)$ which is in the form of (1), where $f\left(x, t_{m}\right)=A y+G$ and $A$ is an $M-1$ square matrix, $G$ is a vector of constants.

BHSDA is L -Stable Block Hybrid Second Derivative Algorithm in Ngwane and Jator [10].

Tables 1 and 2 shows the comparison of exact solution and the mothers H2BM1 and H2BM2 respectively. For example 1.

Table 3 shows the comparison of maximum errors obtained for example 1 using the derived methods and the method in Ngwane and Jator[10]. This shows the superiorly of the derived methods over existing methods.

Figure 1 show the surface plots for the exact solution and Numerical solutions for example 1.

Example 2. Consider the PDE ([14]):

$$
\begin{aligned}
& \frac{\partial y^{2}}{\partial x^{2}}+\frac{\partial y^{2}}{\partial t^{2}}=-32 \pi^{2} \sin (4 \pi x), \quad x \in[0,1] \\
& y( \pm 1, t)=y(x, \pm 1)=0, \quad t>0
\end{aligned}
$$

Table 3: Comparison of maximum errors obtained in different methods for Example 1 at $t=1$.

\begin{tabular}{cccc}
\hline$\kappa$ & H2BM1 & H2BM2 & BHSDA \\
\hline 1 & $1.076 \times 10^{-11}$ & $1.022 \times 10^{-12}$ & $2.64 \times 10^{-6}$ \\
\hline 2 & $1.024 \times 10^{-12}$ & $1.085 \times 10^{-12}$ & $1.32 \times 10^{-6}$ \\
\hline 3 & $1.045 \times 10^{-12}$ & $1.045 \times 10^{-12}$ & $1.32 \times 10^{-6}$ \\
\hline 5 & $1.035 \times 10^{-12}$ & $1.055 \times 10^{-12}$ & $1.32 \times 10^{-6}$ \\
\hline 10 & $1.019 \times 10^{-12}$ & $1.041 \times 10^{-12}$ & $1.32 \times 10^{-6}$ \\
\hline
\end{tabular}




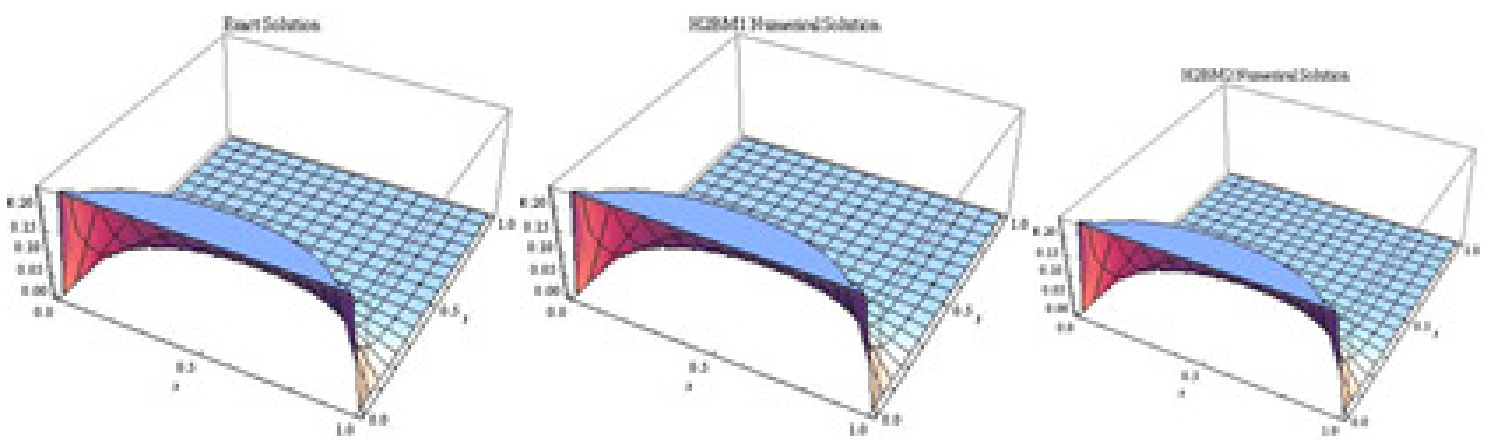

Figure 1: Surface plots for the Exact and numerical solution for Example 2

Table 4: Exact and Numerical solution using H2BM1 for Example 2.

\begin{tabular}{cccc}
\hline$\kappa$ & H2BM1 & H2BM2 & BHSDA \\
\hline $\mathrm{x}$ & Exact & H2BM1 & Error \\
\hline 0.0 & $6.634320126 \mathrm{E}-16$ & $6.634320126 \mathrm{E}-16$ & 0. \\
0.2 & 0.5590169122545 & 0.5590169122520 & $2.51 \mathrm{E}-12$ \\
0.4 & -0.9045084378512 & -0.9045084378511 & $1.00 \mathrm{E}-12$ \\
0.6 & 0.9045084354472 & 0.9045084354462 & $1.00 \mathrm{E}-12$ \\
0.8 & -0.5590169311454 & -0.5590169311421 & $3.37 \mathrm{E}-12$ \\
1.0 & $-4.658833273 \mathrm{E}-16$ & $-4.658833273 \mathrm{E}-16$ & 0. \\
\hline \multicolumn{4}{c}{ for $N=10, x \in[-1,1]$} \\
\end{tabular}

Table 5: Exact and Numerical solution using H2BM2 for Example 2.

\begin{tabular}{cccc}
\hline $\mathrm{x}$ & Exact & $\mathrm{H} 2 \mathrm{BM} 2$ & Error \\
\hline 0.0 & $6.634320126 \mathrm{E}-16$ & $6.634320126 \mathrm{E}-16$ & 0. \\
0.2 & 0.5590169122545 & 0.5590169122589 & $4.49 \mathrm{E}-12$ \\
0.4 & -0.9045084378512 & -0.9045084378577 & $6.52 \mathrm{E}-12$ \\
0.6 & 0.9045084354472 & 0.9045084354428 & $5.53 \mathrm{E}-12$ \\
0.8 & -0.5590169311454 & -0.5590169311433 & $2.12 \mathrm{E}-12$ \\
1.0 & $-4.658833273 \mathrm{E}-16$ & $-4.658833273 \mathrm{E}-16$ & 0. \\
\hline \multicolumn{4}{c}{ for $N=10, x \in[-1,1]$} \\
\end{tabular}

The analytic solution is $y(x, t)=\sin 4 \pi x \sin 4 \pi t$.

Following Ngwane and Jator [10], (38) becomes

$$
\begin{aligned}
& \frac{\mathrm{d} y_{m}^{2}}{\mathrm{~d} x^{2}}=-\frac{\left.y\left(x, t_{m+1}\right)-2 y\left(x, t_{m}\right)+y\left(x, t_{m-1}\right)\right]}{(2 \Delta t)} \\
& -32 \pi^{2} \sin (4 \pi x)
\end{aligned}
$$

$$
y_{m}\left( \pm 1, t_{m}\right)=y_{m}(\mathrm{x}, \pm 1)=0,
$$

where

$$
\begin{gathered}
t_{m}=m \Delta t, \quad m=0,1, \ldots, M ; \\
y_{m}(x) \approx y\left(x, t_{m}\right), y(x)=\left[y_{0}(x), y_{1}(x), \ldots, \mathrm{y}_{M-1}(x)\right]^{T},
\end{gathered}
$$

hence (41) becomes the system $\frac{d^{2} y_{m}(x)}{d \mathrm{x}^{2}}=f\left(x, y_{m}\right)$ which is in the form of (1), where $f\left(x, t_{m}\right)=A y+G$ and $A$ is an $M-1$ square matrix, $G$ is a vector of constants.

BVM and BUM are Boundary Value Methods and the Block Unification Methods in Biala [14].

Tables 4-5 shows the comparison of the exact solution and the methods $\mathrm{H} 2 \mathrm{BM} 1$ and $\mathrm{H} 2 \mathrm{BM} 2$ respectively for example 2. Table 6-7 shows the maximum error and CPU time obtained for different methods. Table 9 shows the maximum error and CPU time obtained in Biala [14].
Table 6: Comparison of maximum errors obtained in different methods for Example 2 at $t=1$

\begin{tabular}{ccccc}
\hline $\mathrm{N}$ & H2BM1 $l_{\infty}$ error & CPU Time & H2BM2 $l_{\infty}$ error & CPU Time \\
\hline 16 & $2.257 \mathrm{E}-7$ & 0.112 & $5.547 \mathrm{E}-7$ & 0.121 \\
32 & $2.787 \mathrm{E}-7$ & 0.898 & $1.712 \mathrm{E}-7$ & 0.871 \\
64 & $8.234 \mathrm{E}-7$ & 2.785 & $2.337 \mathrm{E}-7$ & 2.662 \\
128 & $3.114 \mathrm{E}-7$ & 11.211 & $4.785 \mathrm{E}-7$ & 12.009 \\
256 & $2.779 \mathrm{E}-7$ & 31.812 & $1.112 \mathrm{E}-7$ & 30.101 \\
\hline
\end{tabular}

Table 7: Comparison of maximum errors obtained in different methods for Example 2 at $t=1$

\begin{tabular}{ccccc}
\hline $\mathrm{N}$ & $\mathrm{BVM} l_{\infty}$ error & CPU Time & $\mathrm{BUM} l_{\infty}$ error & CPU Time \\
\hline 16 & $9.662 \mathrm{E}-0$ & 0.483 & $1.251 \mathrm{E}-1$ & 0.531 \\
32 & $2.582 \mathrm{E}-2$ & 1.235 & $2.578 \mathrm{E}-2$ & 1.031 \\
64 & $6.433 \mathrm{E}-3$ & 5.358 & $6.459 \mathrm{E}-3$ & 5.516 \\
128 & $1.607 \mathrm{E}-3$ & 43.641 & $1.607 \mathrm{E}-3$ & 46.923 \\
256 & $2.000 \mathrm{E}-0$ & 512.843 & $4.016 \mathrm{E}-4$ & 532.657 \\
\hline
\end{tabular}

This show that the derived methods performs accurately, superiorly and affluently in terms of the computer time, and errors obtained in examples 2 .

Figure 2 shows the surface plots for the exact and Numerical solution for examples 2 .

\section{Example 3.}

We consider the PDE (Jator [15]).

$$
\begin{aligned}
& \frac{\partial y^{2}}{\partial t^{2}}+\frac{\partial y^{2}}{\partial x^{2}}=\sin (y), \quad x \in[-3,3] \\
& y(x, 0)=4 \arctan \left(e^{\frac{x}{\sqrt{1-c^{2}}}}\right) \text {, } \\
& y_{t}(x, 0)=-\frac{4 c e^{\frac{x}{\sqrt{1-c^{2}}}}}{\sqrt{1-c^{2}}\left(1+e^{2 \frac{x}{\sqrt{1-c^{2}}}}\right)}, \quad 0<t<1
\end{aligned}
$$

The analytic solution is $y(x, t)=4 \arctan (\operatorname{sech}(x) t), c$ is velocity of the wave. The problem is solved for $c=0.5, \Delta t=0.125$ Following Ngwane and Jator [10], (38) becomes

$$
\begin{aligned}
& \frac{\mathrm{d} y_{m}^{2}}{\mathrm{~d} x^{2}}=-\frac{\left.y\left(x, t_{m+1}\right)-2 y\left(x, t_{m}\right)+y\left(x, t_{m-1}\right)\right]}{(2 \Delta t)}+\sin \left(\mathrm{y}_{\mathrm{m}}\right)(43) \\
& y_{m}(x, 0)=4 \arctan \left(e^{\frac{x}{\sqrt{1-c^{2}}}}\right), \\
& y_{m t}(x, 0)=-\frac{4 c e^{\frac{x}{\sqrt{1-c^{2}}}}}{\sqrt{1-c^{2}}\left(1+e^{2 \frac{x}{\sqrt{1-c^{2}}}}\right)}, \quad 0<t<1
\end{aligned}
$$



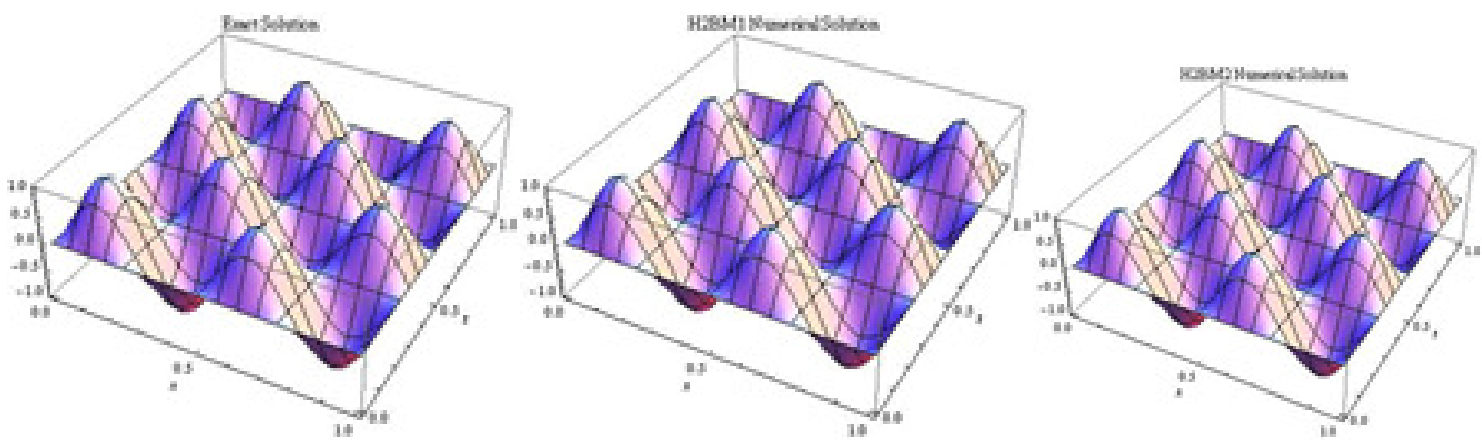

Figure 2: Surface plots for the Exact and numerical solution for Example 3
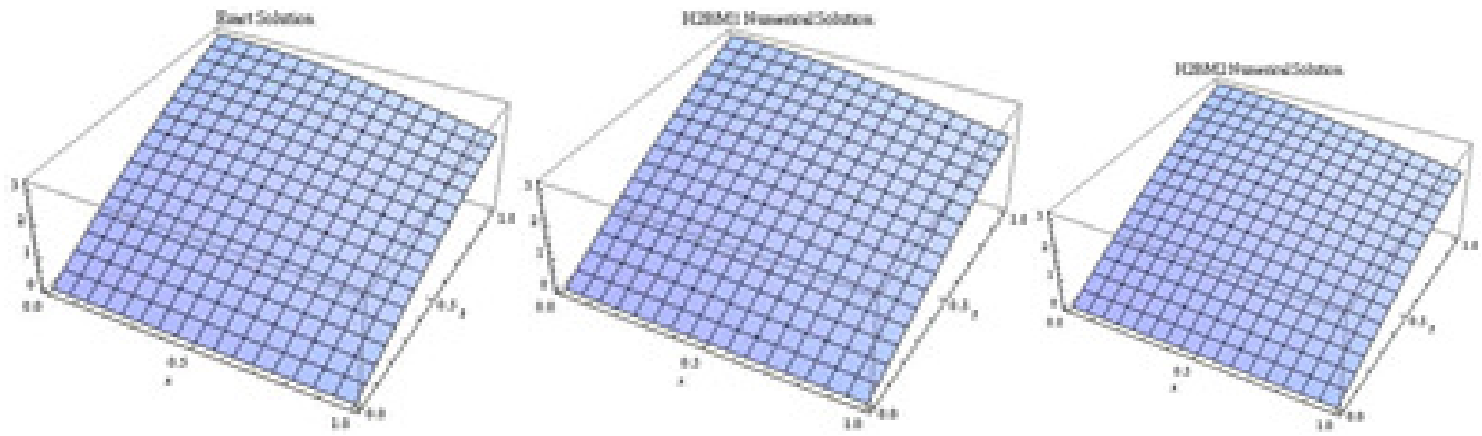

Figure 3: Surface plots for the Exact and numerical solution for Example 3

Table 8: Exact and Numerical solution using different methods for Example 3

\begin{tabular}{ccccc}
\hline $\mathrm{x}$ & Exact & H2BM1 & H2BM2 & SBVM \\
\hline 0.125 & 0.12195641127 & 0.12195641122 & 0.12195641178 & 0.121956 \\
0.25 & 0.11346954831 & 0.11346954852 & 0.11346954877 & 0.113469 \\
0.375 & 0.10557254177 & 0.10557254144 & 0.10557254129 & 0.105573 \\
0.5 & 0.09822454418 & 0.09822454411 & 0.09822454412 & 0.0982256 \\
0.625 & 0.09138842135 & 0.09138842122 & 0.09138842129 & 0.0913892 \\
0.75 & 0.08502738529 & 0.08502738541 & 0.08502738557 & 0.0850284 \\
0.875 & 0.07910885264 & 0.07910885215 & 0.07910885213 & 0.0791101 \\
1.00 & 0.07360212510 & 0.07360212548 & 0.07360212544 & 0.0736035 \\
\hline
\end{tabular}

Table 9: Approximate and Numerical solution for Example 3

\begin{tabular}{cccc}
\hline $\mathrm{x}$ & H2BM1 Error & H2BM2 Error & SBVM Error \\
\hline 0.125 & $7.2 \mathrm{E}-10$ & $5.1 \mathrm{E}-10$ & $1.30 \mathrm{E}-7$ \\
0.25 & $2.1 \mathrm{E}-11$ & $4.6 \mathrm{E}-10$ & $2.94 \mathrm{E}-7$ \\
0.375 & $3.3 \mathrm{E}-11$ & $4.8 \mathrm{E}-10$ & $4.51 \mathrm{E}-7$ \\
0.5 & $7.2 \mathrm{E}-11$ & $8.0 \mathrm{E}-10$ & $6.49 \mathrm{E}-7$ \\
0.625 & $1.4 \mathrm{E}-10$ & $5.5 \mathrm{E}-10$ & $8.41 \mathrm{E}-7$ \\
0.75 & $1.2 \mathrm{E}-10$ & $3.7 \mathrm{E}-10$ & $1.03 \mathrm{E}-6$ \\
0.875 & $4.9 \mathrm{E}-10$ & $3.3 \mathrm{E}-10$ & $1.25 \mathrm{E}-6$ \\
1.00 & $3.8 \mathrm{E}-10$ & $7.7 \mathrm{E}-10$ & $1.44 \mathrm{E}-6$
\end{tabular}

where

$t_{m}=m \Delta t, \quad m=0,1, \ldots, M ; y_{m}(x) \approx y\left(x, t_{m}\right), y(x)=[$ $\left.y_{0}(x), y_{1}(x), \ldots, \mathrm{y}_{M-1}(x)\right]^{T}$,

hence (43) becomes the system $\frac{d^{2} y_{m}(x)}{d x^{2}}=f\left(x, y_{m}\right)$ which is in the form of (1).

where $f\left(x, t_{m}\right)=A y+G$ and $A$ is an $M-1$ square matrix, $G$ is a vector of constants.

SBVM is symmetric boundary value method in Jator [15].
Table 8 shows the exact and numerical solution using the different methods for example 3 while Table 9 shows error obtained for example 3. Figure 3 shows the surface plots for the Exact and numerical solutions of H2BM1 and H2BM2 for Example 3

\section{Conclusion}

The development of some numerical schemes has been proposed in this work. This was developed via the interpolation and collocation techniques using power series function as trial solutions. The methods were effectively illustrated some partial differential equations (PDE) and the results obtained were accurate. The analysis of the new methods showed that all satisfy the properties of numerical methods for the solution of differential equations. Namely, Consistency, Zero- Stability, Continuity and convergence.

\section{References}

[1] L. Brugnano, L. \& D. Trigiante, "Solving Differential Problems by Multistep Initial and Boundary Value Methods", Gordon and Breach Science Publishers, Amsterdam, (1998).

[2] P. Onumanyi, U.W. Sirisena. \& S.N Jator, "Continuous finite difference approximations for solving differential equations", International. Journal of. Computer Mathematics. 72 (1999) 15.

[3] P. Onumanyi, D. O. Awoyemi, S. N. Jator \& U. W. Sirisena, "New linear multistep methods with continuous coefficients for first order initial value problems", 19 Journal of Nigeria Mathematics Society 13, (1994) 37.

[4] S. N. Jator "On the Hybrid Method with Three-Off Step Points for Initial Value Problems", International Journal of Mathematical Education in Science and Technology, 41 (2010) 110 
[5] S. N. Jator "Trigonometric symmetric boundary value method for oscillating solutions including the sine-Gordon and Poisson equations", Cogent Mathematics 3 (2016) 1271.

[6] S. O. Fatunla, "Block Methods for second-order IVP", International Journal of Mathematics 55 (1999).

[7] Y. Yusuph \& P. Onumanyi; "New multiple FDM through multistep collocation" Proceedings of the conference, National Mathematical Centre, Abuja (2005).

[8] Siraj-ul-Islam, Imran Aziz \& Bozidar Sarler, "The numerical solution of second-order boundary-value problems by collocation method with the Haar wavelets", Mathematical and Computer Modelling 52 (2010) 1577.

[9] J. Adewale, A. Olaide. \& J. Sunday, "Continuous Block Method for the Solution of second rder Initial Value Problems of Ordinary Diff. Equation" International Journal of Pure and Applied mathematics 82 (2013) 405.

[10] F. F. Ngwane \& S. N. Jator, " $L$-Stable Block Hybrid Second Derivative Algorithm for Parabolic Partial Differential Equations", American Journal of Computational Mathematics 4 (2014) 87.
[11] J. Vigo-Aguiar \& H. Ramos, "A family of A-Stable Collocation Methods of Higher Order for Initial-Value Problems", IMA Journal of Numerical Analysis 27 (2007) 798.

[12] J. R, Cash "Two New Finite Difference Schemes for Parabolic Equations", SIAM Journal of Numerical Analysis 21 (1984) 433.

[13] S. N. Jator \& J. Li, "An algorithm for Second Order Initial and Boundary Value Problems" Numerical Algorithm (2012) 59.

[14] T. A. Biala, "Computational Study of the Boundary Value Methods and the Block Unification Methods ", Abstract and Applied Analysis (2012) Article ID 8465103, 14 pages

[15] P. Henrici, "Discrete Variable Methods in Ordinary Differential Equations", John Wiley, New York, 1962.

[16] J. D. Lambert, "Computational Methods in Ordinary Differential Equations", John Wiley and Sons, New York, NY, USA, 1973.

[17] M. I. Modebei, R. B. Adeniyi, S. N. Jator \& H. C. Ramos "A block hybrid integrator for numerically solving fourth-order Initial Value Problems", Applied Mathematics and Computation 346 (2019) 680. 